\title{
ORCHIDACEAS DO ESTADO DO MARANHÃO, BRASIL ${ }^{1}$
}

\author{
Manoela F. F. da SILVA², João Batista F. da SILVA ${ }^{3}$, Jacqueline M. FEILER ${ }^{4}$
}

RESUMO - O Estado do Maranhão faz parte da Amazônia Legal e tem sido um dos mais atingidos pelos projetos impactadores do meio ambiente, causando sérios riscos de extinção de espécies vegetais e animais, algumas, ainda desconhecidas pela ciência. Com o objetivo de conhecer a flora orquídica daquele Estado foram conduzidos estudos ao longo de aproximadamente 12 anos, registrando-se a ocorrência de 103 diferentes espécies de Orchidaceae. Os resultados demonstraram a grande riqueza em espécies nesta família, e o quanto ela era desconhecida, uma vez que antes deste estudo, conheciase pela literatura, apenas dez espécies ocorrendo no Estado do Maranhão.

PALAVRAS CHAVE: Orchidaceae, Amazônia Legal, Diversidade

\section{Checklist of Orchidaceae from State of Maranhão, Brazil.}

ABSTRACT - The Maranhão State is part of "Amazônia Legal", and has been one of the most affected by the impacts of projects to environment, and leading to extintion many species of plants and animals, some of them not discovered by science. A study with the orchids flora of Maranhão state for about 12 years registered the occurence of 103 different species of Orchidaceae. These results show a great variety of the species in this this family and how much it was unknown, since, before this study, of only 10 different species of Orchidaceae, were cited the in Brazilian State of Maranhão.

KEY WORDS: Orchidaceae, Legal Amazon, Diversity

\section{INTRODUÇÃO}

O Estado do Maranhão faz parte da Amazônia Legal, e como tal, nas décadas de 70 e 80 foi alvo de grandes incentivos em forma de financiamentos para grandes projetos, em especial de agropecuária. As ações destes projetos são grandes impactadoras do meio ambiente, causando a destruição da flora e fauna local, tanto a nivel especifico como de comunidades desses organismos, desestruturando-as, causando alteração no balanço energético, na cadeia alimentar, na ciclagem de nutrientes, entre outros danos ambientais incalculáveis. As florestas ao serem derrubadas e queimadas pode, ainda, causar o desaparecimento de muitas espécies vegetais antes mesmo de serem conhecidas à luz da ciêncía. As Orchidaceae são exemplos típicos deste fato, por serem plantas normalmente de hábito epifitico, sendo pouco coletadas devido à maioria não ser alcançada nas coletas gerais, razão pela qual muitas espécies não figuram nos acervos das coleções científicas e, assim, não são citadas na literatura.

Antes de iniciar as pesquisas de campo deste projeto era citado na bibliografia a ocorrência de apenas 10 espécies de Orchidaceae para aquele Estado (Pabst, 1975, 1977). Após os primeiros levantamentos de campo verificou-se que a flora orquídica da região era muito mais rica, porém, ainda desconhecida.

Este trabalho representa o resultado

\footnotetext{
Projeto Integrado CNPq/Processo: 521626/93-5.

Profa. Visitante da Fac, de Ciênc. Agrảias do Pará e Bolsista do Dept. Botânica - MPEG/CNPq Orquidólogo autônomo, participante e colaborador do Projeto.

4 Bolsista de Iniciação Cientifica do MPEG/CNPq.
} 
dos levantamentos da flora orquidica do Estado do Maranhão, aqui apresentado na forma de uma listagem das Orchidaceae registradas para a região.

\section{MATERIAL E MÉTODOS}

\section{REGIÃO DE ESTUDO}

O Estado do Maranhão situa-se numa região de transição entre várias provincias fitogeográficas, limitando-se a leste com a região nordeste, a oeste com a Amazônia, ao norte com o oceano Atlântico e, ao sul com a região Centro Oeste do Brasil. Esta posição geográfica confere-lhe características ecológicas e floristicas bem peculiares. A maior parte de seu território está situada na província amazônica com uma larga faixa de transição entre a floresta amazônica propriamente dita e a caatinga, localizada entre as bacias dos rios Grajaú e Pindaré. A partir da região central do Estado, na área limitada pelos rios Grajaú e Itapecuru, situa-se a zona dos cocais, cuja vegetação primitiva era de floresta, mesclada com palmeiras "babaçu"Attalea speciosa Mart. ex Spreng. Com a devastação da floresta, restaram apenas as palmeiras, dai essa região ser conhecida atualmente como Zona dos Cocais. Ao sul, entre os rios Itapecuru e Parnaiba, a vegetação é tipica de cerrado, intercalada com vegetação da transição com a caatinga, onde são freqüentes os mananciais de águas perenes chamados brejos. $\mathrm{O}$ extremo sul do Estado é coberto por vegetação de cerrado, onde localizam-se as elevações, e nascem os principais rios.

A diversidade de ambientes e de tipos vegetacionais correspondem a uma singular riqueza floristica, principalmente em áreas como os brejos, que possuem uma caracterização edafo-ecológica bem diferenciada.

Na zona central-sul, situam-se os brejos formadores das bacias dos rios Corda e das Flores, ambos afluentes da margem direita do rio Mearim, um dos principais rios do Estado. Os brejos são fontes de água perene e correm num vale arenoso, cortando o cerrado até desaguarem num dos rios principais. A calha desses rios contém áreas cultivadas há muitos anos e por isso não há mais vegetação primitiva, restando apenas as palmeiras de "babaçu", que servem de substrato à espécies do gênero Catasetum, em altas concentrações, e outras epífitas. Este fato despertou a nossa atenção para um estudo mais detalhado das espécies ocorrentes nos brejos, resultando na descoberta de várias espécies até então desconhecidas e que estão sendo analisadas por especialistas.

Na Figura 1 estão assinaladas as áreas de ocorrência e de coleta das espécies relacionadas neste trabalho.

\section{COLETA E ESTUDO DO MATERIAL BOTÂNICO}

Os espécimes de Orchidaceae, quando coletados em estado fértil eram registrados e identificados no campo (quando tratava-se de espécies conhecidas e de fácil identificação). Quando isso não era possível procedia-se à coleta do material para identificação por comparação com material herborizado já identificado por especialistas, com ilustrações e com as descrições da literatura 


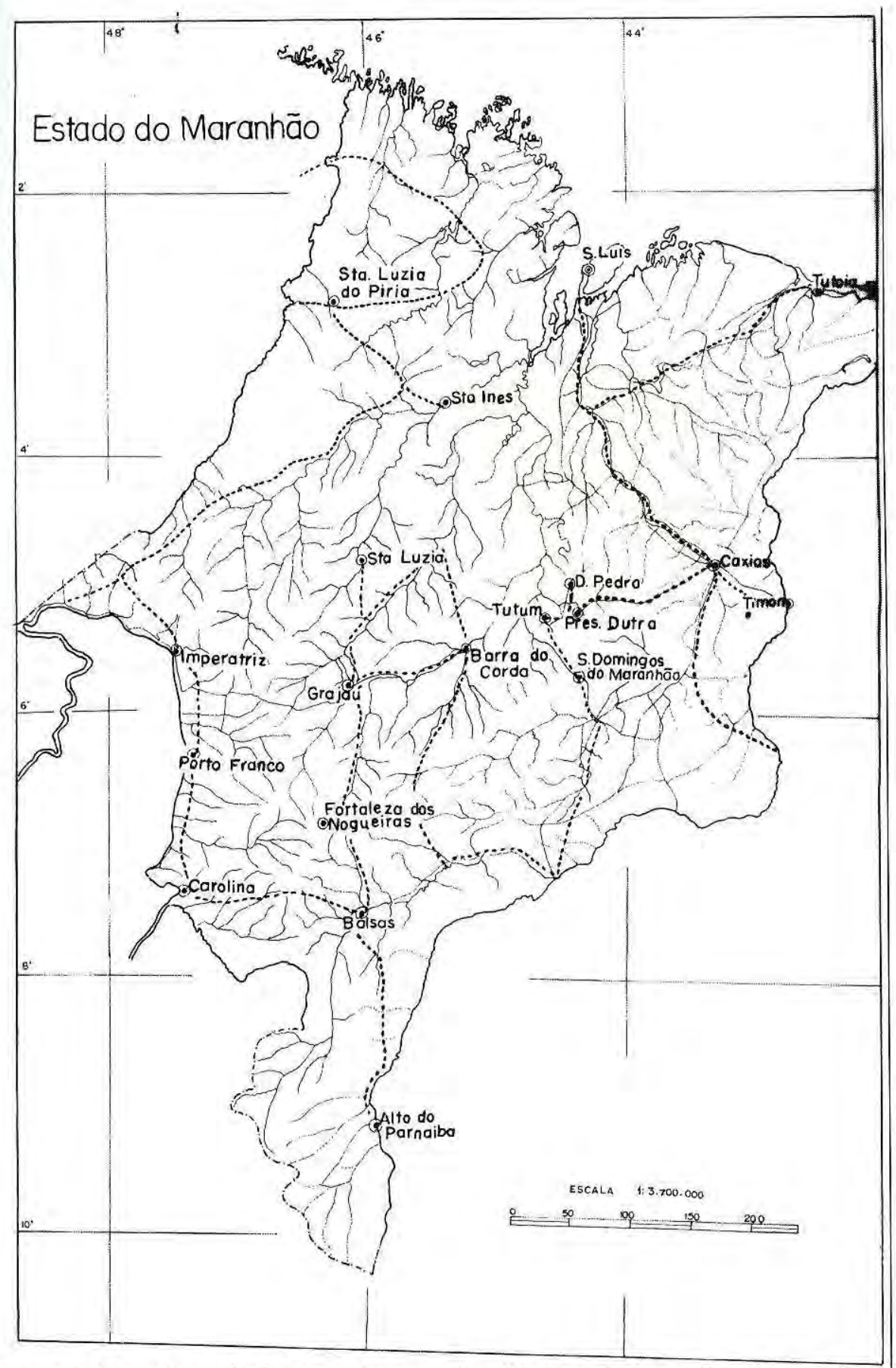

Figura 1. Mapa do estado do Maranhão com as localidades onde foram efetuadas as coletas de material e dados de campo. 
Tabela 1. Distribuição das espécies de Orchidaceae por habitat, no Estado do Maranhão.

TI (Terrestre de campos inundáveis)

TC (Terrestre de vegetação de cerrado)

EC (Epífita de cerrado)

ER (Epífita de floresta ribeirinha)

EA (Epífita de floresta alagada)

ECa (Epífita de campina)
TF (Terrestre de terra-firme)

El (Epífita de igapó)

EF (Epífita de floresta de terra-firme)

EG (Epífita de floresta de galeria)

EU (Epífita de floresta úmida)

ECr (Epífita de campo rupestre)

\section{HABITATS}

\section{ESPÉCIES}

Aganisia pulchella Lindl.

Aspasia variegata Lindl.

Bletia catenulata Ruiz \& Pavon.

Brassavola gardneri Cogn.

Brassia caudata Lindl.

Bulbophyllum insectiferum Barb.Rodr.

Bulbophyllum setigerum Lindl.

Campylocentrum amazonicum Cogn.

Campylocentrum micranthum Rolfe

Catasetum albovirens Barb. Rodr.

Catasetum barbatum Lindl.

Catasetum carolinianum Mir. \& Lac.

Catasetum discolor Lindl.

Catasetum galeritum Rchb.f.

Catasetum macrocarpum L.C. Rchb.

Catasetum sp. 1

Catasetum sp. 2

Catasetum sp. 3

Catasetum sp. 4

Catasetum sp. 5

Catasetum sp. 6

Catlleya nobilior Rchb. f.

Cleistes paludosa Rchb. $\mathrm{f}$.

Cleistes rosea Lindl.

Cyrtopodium andersonii (Lamb.) R.Br.

Cyrtopodium virescens R. f. et. Warm

Dichaea picta Rchb. F.

Dimeranda emarginata (G. M.) Hoehne

Encyclia fragrans (SW) Dressl.

Encyclia granitica Schltr.

Encyclia linearifolioides (Kr.) Hoehne

Epidendrum amblostomoides Hoehne

Epidendrum ciliare L.

Epidendrum difforme Jacq.

Epidendrum imatophyllum Lindl.

Epidendrum nocturnum Jacq.

Epidendrum purpurascens Focke

Epidendrum rigidum Jacq.

Epidendrum schomburgkii Lindl.

Epidendrum strobiliferum Rchb.f.

Epidendrum viviparum Lindl.

Eulophia alta (L.) Fawc.et. Rendle

Galeandra sp.

Galeandra styllomisantha (Vell.) Hoehne $x$

Gongora nigrita Lindl.

$x$

X

TI TF TC EI EC EF ER EG EA EU Eca ECr

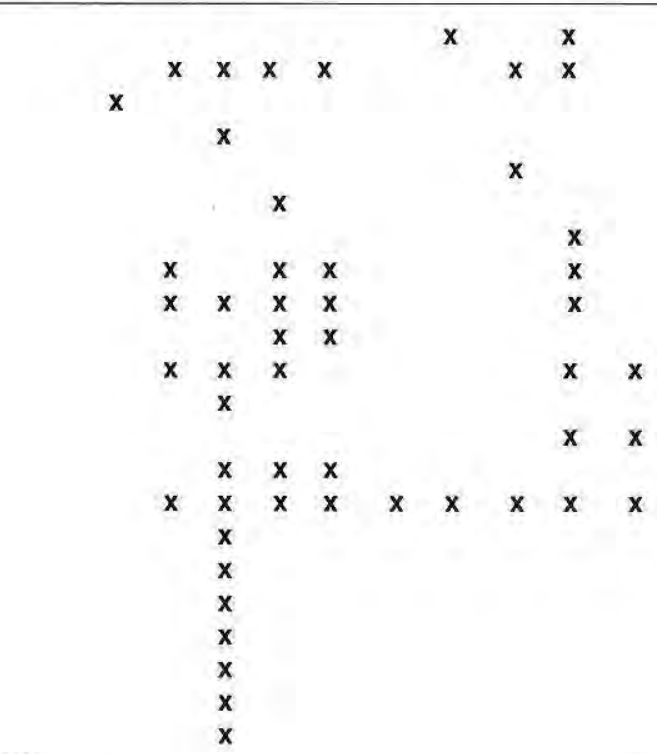

$\mathbf{x}$

$\mathbf{X}$

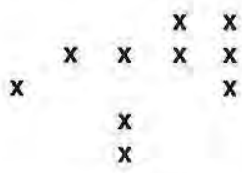

$x \quad x$

$x$

X

x $\times \quad \times \quad x$

$x \quad x \quad x$

$x \quad x$

$x \quad x$

$x$ 
Cont. Tabela 1.

Gongora quinquenervis Ruiz \& Pavon

Habenaria obtusa Lindl.

Habenaria pratensis (Lindl.) Rchb.f.

lonopsis satyrioides Rchb.f.

x

lonopsis utricularioides Lindl.

Lepanthes brasiliensis Pabst

Lepanthes helicocephala Rchb.f.

Leucohyle brasiliensis Schltr.

Leucohyle mutica (Rchb.f. \& W.) Schltr

Lockhartia goyazensis Rchb.f.

Lockhartia lunifera Rchb.f.

Macradenia rubescens B. Rodr.

Maxillaria alba (Hook) Lindl.

Maxillaria camaridii Rchb.f.

Maxillaria notylioglossa Rchb.f.

Maxillaria rufescens Lindl.

Maxillaria superflua Rchb.f.

Maxillaria uncata Lindl.

Maxillaria vilosa (Barb. Rodr.) Cogn.

Mormodes sp

Notylia aromatica Baker ex. Lindl.

Notylia lirata Moore

Notylia wullschlaegeliana Focke

Octomeria grandiflora Lindl.

Oeceoclades maculata (Lind.) Lindl.

Oncidium baueri Lindl.

Oncidium cebolleta Swarez (Jacq) Sw.

Oncidium fuscopetalum (Hohene) G.

Oncidium macropetalum Lindl

Oncidium morenoi Dodson \& Luer

Oncidium nanum Lindl.

Orleanesia amazonica Barb. Rodr.

Orleanesia yauaperyensis Barb. Rodr.

Ornithocephalus gladiatus Hook

Peristeria guttata Knowl

Pleurothallis modesta Cogn.

Pleurothallis pruinosa Lindl.

Polystachya concreta (Jacq) Garay

Polystachya foliosa Rchb.f.

Polystachya stenophylla Schltr.

Psygmorchis glossomistax Rchb.f.

Psygmorchis pusilla (L.) Dod. \& Dres.

Quekettia microscopica Lindl.

Reichenbachanthus reflexus (L.) Brad.

Rodriguezia lanceolata Lood.

Sacoila lanceolata (Aubl.) Garay

Scaphyglottis amethystina (Rchb.f.)

Scaphyglottis modesta Schltr.

Scaphyglottis sickii Pabst.

Schomburgkia gloriosa Rchb.f.

Sobralia macrophylla Rchb.f.

Sobralia sessilis Lindl.

Solenidium lunatum Schltr.

Stanhopea grandiflora Lindl.

Trigonidium acuminatum Batem.

Trigonidium tenue Lodd.

Vanilla palmarum Lindl.

Zigosepalum labiosum (L.C.Rich) Gar.
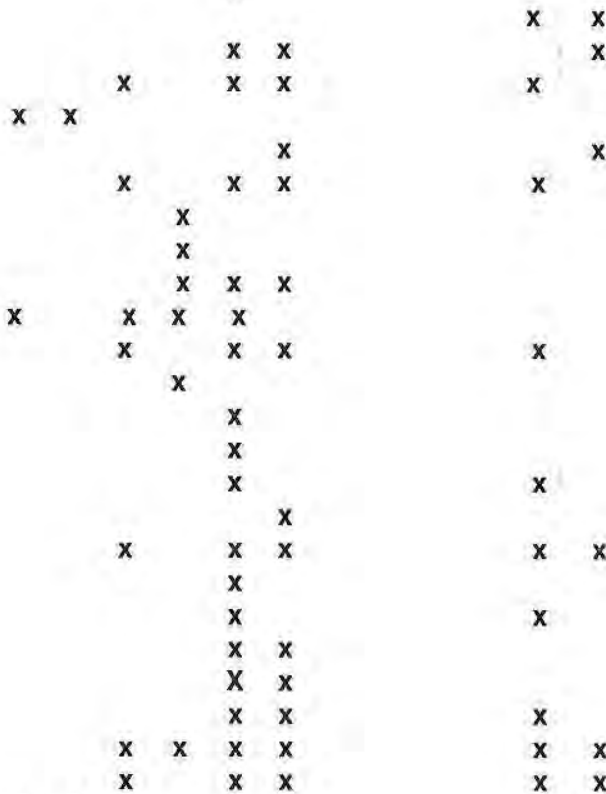

$\mathrm{x}$

X

X

x $x$

\begin{tabular}{|c|c|c|c|c|c|}
\hline \multirow[t]{3}{*}{$x$} & $x$ & $x$ & $x$ & $x$ & $x$ \\
\hline & & $x$ & & 1 & \\
\hline & & $x$ & $\mathbf{X}$ & & $x$ \\
\hline \multirow[t]{2}{*}{$x$} & $x$ & $x$ & $x$ & $x$ & $x$ \\
\hline & & & $x$ & $x$ & \\
\hline \multirow[t]{4}{*}{ X } & & & $x$ & $\mathrm{x}$ & \\
\hline & & $x$ & $x$ & $x$ & $x$ \\
\hline & & $x$ & $x$ & & \\
\hline & & & $x$ & & \\
\hline \multirow[t]{2}{*}{$\mathbf{X}$} & $x$ & $x$ & $x$ & $x$ & $x$ \\
\hline & & $x$ & $x$ & & \\
\hline 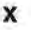 & & & $x$ & & \\
\hline
\end{tabular}


especializada. Quando era impossivel a identificação por nossa equipe, por se tratar, provavelmente, de um novo Táxon, após a tentativa de identificação e realizada a respectiva ilustração em nanquim ou aquarela, parte do material foi fixado em álcool a 70\%, principalmente a inflorescência, para posteriormente ser examinado por especialista. Finalmente procedeu-se a herborização e registro na coleção do herbário do Museu Paraense Emilio Goeldi-(PA).

Como já foi citado, no caso de ser espécie muito comum ou de um único espécime e de fácil identificação, o mesmo não foi coletado, não possuindo assim número de registro de herbário.

As principais obras consultadas para a identificação dos espécimes foram: Cogniaux (1896; 1902; 1906), Hoehne $(1942 ; 1945 ; 1949 ; 1953)$, Dunsterville \& Garay (1972-1976), Pabst \& Dungs $(1975 ; 1977) \mathrm{e}$ Dressler (1993).

\section{RESULTADOS}

Foram catalogadas para o Estado do Maranhão 103 espécies da familia Orchidaceae distribuidas em 48 gêneros diferentes. Dessas, sete são possivelmente espécies novas para a ciência e ainda estão em estudo.

A despeito da região estudada ser muito grande e envolver muitos ecossistemas diferentes e uma ampla diversidade de habitats, a maioria das espécies é de habito epifítico nas florestas ribeirinhas, floresta de terra firme e floresta de igapó, conforme pode ser verificado tabela 1 , enquanto que as espécies terrestres são em número reduzido, enquadrando-se no padrão geral das espécies de Orchidaceae das regiões tropicais.

A seguir são apresentadas as espécies de que trata este trabalho, seguidas de informações sobre a distribuição geográfica e o habitat.

ESPÉCIE: Aganisia pulchella Lindl. MG 133.532

HABITAT: Floresta ribeirinha, campina. DISTRIBUIÇÃO: Guianas, Brasil: AM, MA, PA

ESPÉCIE: Aspasia variegata Lindl. MG 145. 496

HABITAT: Floresta de terra-firme, floresta ribeirinha, campina, igapó e cerrado.

DISTRIBUIÇÃO: Trinidad, Venezuela, Guiana, Suriname, Colômbia e Brasil: AM, MA, PA, RO, RR, TO

ESPÉCIE: Bletia catenulata Ruiz et. Pav. MG 142.492

HABITAT: Cerrado

DISTRIBUIÇÃO: Peru, Brasil: MA, TO, SP, MG, GO, DF, MT

ESPÉCIE: Brassavola gardineri Cogn. MG 141.266

HABITAT: Floresta de galeria de cerrado DISTRIBUICAO: Brasil: AP, PA, GO

ESPÉCIE: Brassia caudata Lindl. HABITAT: Epifita de floresta úmida DISTRIBUIÇÃO: América Central, México, Brasil: MA, PA, RO

ESPÉCIE: Bulbophyllum insectiferum Barb. Rodr. MG 112.423

HABITAT: Floresta de terra firme DISTRIBUIÇĀO: Brasil: DF, GO, MG, MA

ESPECIE: Bulbophyllum setigerum Lindl., HABITAT: Campina

DISTRIBUIÇÃO: Guiana Francesa, 
Suriname, Guiana, Venezuela e Brasil: MA, MT, PA

ESPÉCIE: Campylocentrum amazonicum Cogn. MG 138.871

HABITAT: Floresta de terra-firme, capoeira, campina e pomares.

DISTRIBUIÇÃO: Venezuela, Guiana, Brasil: AC, AM, MA, PA

ESPÉCIE: Campylocentrum micranthum (Lindl.) Rolfe. MG 145.540

HABITAT: Epifita de florestas e campos.

ESPÉCIE: Catasetum albovirens Barb. Rodr. MG 133.547

HABITAT: Floresta de terra-firme, floresta ribeirnha.

DISTRIBUIÇÃO: Brasil: AM, MA, PA

ESPÉCIE: Catasetum barbatum Lindl. MG 138.982

HABITAT: Floresta de terra-firme, Igapó, campina, cerrado e campo rupestre DISTRIBUIÇÃO: Peru, Bolivia, Equador, Venezuela, Guiana, Suriname, Brasil: MA, PA, TO, AM, RR, RO, PI, CE

ESPÉCIE: Catasetum carolinianum Miranda \& Lacerda. MG 145.988

HABITAT: Epifita em palmeira na terra firme, cerrado

DISTRIBUIÇÃO: Brasil: MA, TO.

ESPÉCIE: Catasetum discolor Lindl. MG 145.523

HABITAT: Igapó, campina e campo rupestre

DISTRIBUIÇÃO: Colômbia, Guiana, Suriname, Venezuela, Brasil: AM, AP, BA, CE, ES, MA, PA, PE RO

ESPÉCIE: Catasetum galeritum Rchb.f. MG 138.849

HABITAT: Área de transição entre vegetação rupestre e floresta alta
DISTRIBUIÇÃO: Brasil: AM, PA, MA, TO

ESPÉCIE: Catasetum macrocarpum L. C. Rich, ex Kunth. MG 147.424

HABITAT: Floresta de terra-firme, caatinga arenosa, floresta hidrófila, floresta de galeria.

DISTRIBUIÇÃO: América Central, Trinidad, Tobago, Peru, Equador, Guiana, Suriname, Guiana Francesa, Venezuela, Colômbia, Brasil: AM, AP, BA, MA, MT, PA, RJ, TO

ESPÉCIE: Cattleya nobilior Rchb.f. MG 356

HABITAT: Cerrado.

DISTRIBUIÇÃO: Brasil: AM, GO, MA, MT

ESPÉCIE: Cleistes paludosa Rchb.f. MG 83.171

HABITAT: Campina

DISTRIBUIÇÃO: Guianas, Brasil: MA, MA, MT

ESPÉCIE: Cleistes rosea Lindl. MG 53.635

HABITAT: Campina, cerrado.

DISTRIBUIÇÃO: Colômbia, Equador, Guiana, Panamá, Peru, Suriname, Venezuela, norte do Brasil, até o Maranhão.

ESPÉCIE: Cyrtopodium andersonii ( Lam. Ex Andr.) Barb. Rodr. MG 98104

HABITAT: Campina e campos rupestres

DITRIBUIÇÃO: Indias Ocidentais, Venezuela, Guianas, Brasil: AP, DF, MG, PA, PE, RO, RR, RJ, SC, MA

ESPÉCIE: Cyrtopodium virescens Rchb.f. et Warm. MG 146.951

HABITAT: Campina, cerrado

DISTRIBUIÇÃO; Brasil: DF, GO, MA, MG, PA, TO

ESPÉCIE: Dichaea picta Rchb.f. MG 151.039

HABITAT: Epifita de floresta úmida 
DISTRIBUIÇÃO: Brasil: AM, MA, PA

ESPÉCIE: Dimeranda emarginata (Meyer) Hoehne. MG 149.865

HABITAT: Epifita de vegetação aberta. DISTRIBUIÇÃO: Peru, Venezuela, Brasil: AP, BA, ES, MA, PA, PE, SE

ESPÉCIE: Encyclia fragrans (Sw.) Lemée. MG 145.507

HABITAT: Floresta de galeria, floresta de terra-firme, igapó, campina, floresta ribeirinha, campo rupestre, cerrado e caatinga arenosa

DISTRIBUIÇÃO: Desde o México, Flórida e Índias Ocidentais, Bolívia, Peru, Brasil: AM, PA, PE, SE, BA, MA, MG, MS, RJ, PR, RS, SC, SP

ESPÉCIE: Encyclia granitica Schltr. MG 146.068

HABITAT: Campina baixa de areia branca na margem esquerda.

DISTRIBUIÇÃO: Guiana, Suriname e Brasil: AP, MA, PA

ESPÉCIE: Encyclia linearifolioides (Krenzl.) Hoehne. MG 133533

HABITAT: Floresta de terra-firme, floresta ribeirinha

DISTRIBUIÇÃO: Brasil: GO, MA, MG, PA, TO,

ESPECIE: Epidendrum amblostomoides Hoehne. MG 138.889

HABITAT: Cerrado

DISTRIBUICAO: GO, MA

ESPÉCIE: Epidendrum ciliare Linn. MG 147.849

HABITAT: Igapó.

DISTRIBUIÇÃO: México, América Central, Venezuela, Colômbia, Guianas, Equador, Peru.e Brasil

ESPÉCIE: Epidendrum difforme Jacq.
MG 139.103

HABITAT: Floresta úmida

DISTRIBUIÇÃO: AM, AP, MA, PA, RO, RR, TO, até o Sul do Brasil

ESPÉCIE: Epidendrum imatophyllum Lindl. MG 148.497

HABITAT: Floresta ribeirinha.

DISTRIBUIÇÃO: Desde México e América Central até Trinidad, Paraguai, Peru, Guianas Brasil: AM, AP, BA, GO, MA, MG, MT, PA, PB, PE, RR

ESPÉCIE: Epidendrum nocturnum Jacq. MG 146.052

HABITAT: Floresta de terra-firme, floresta ribeirinha, campina, campo rupestre, cerrado

DISTRIBUIÇÃO: Flórida, México, América do Sul, Brasil: AC, AM, AP, DF, ES, GO, MA, MG, MT, PA, PE, PR, RS, $\mathrm{SC}, \mathrm{SP}$

ESPÉCIE: Epidendrum purpurascens Focke. MG 135.361

HABITAT: Campo rupestre.

DISTRIBUIÇÃO: Norte da América do Sul, América Central e Brasil: AM, AP, MA, PA

ESPÉCIE: Epidendrum rigidum Jacq. MG 135.362

HABITAT: Floresta úmida

DISTRIBUIÇÃO: AC, AM, AP, MA, PA, RO, RR,

ESPÉCIE: Epidendrum schomburgkii Lindl. MG148.495

HABITAT: Floresta úmida

DISTRIBUIÇÃO: Brasil: toda a Amazônia.

ESPÉCIE: Epidendrum sculptum Rchb. f. MG 138.854

HABITAT: Floresta ribeirinha, floresta de terra-firme, igapó e campina

DISTRIBUIÇÃO: Honduras, Costa Rica, 
Panamá, Colômbia, Venezuela, Guiana, Suriname e Brasil:AM, MA, PA

ESPÉCIE: Epidendrum strobiliferum Rchb. f.

HABITAT: Áreas abertas como campina, pomar e igapó

DISTRIBUIÇÃO: Brasil: AC, AM, GO, MA, MG, MT, PR, RR, SP

ESPÉCIE: Epidendrum viviparum Lindl. MG 139.444

HABITAT: Epifita de floresta aberta tipo caatinga

DISTRIBUIÇÃO: Guianas e Brasil: MA, MT, PA

ESPÉCIE: Galeandra sp. MG 147.011

HABITAT: Cerrado.

DISTRIBUIÇÃO: MA, PA

ESPÉCIE: Galeandra styllomisantha (Vell.) Hoehne. MG 149.896

HABITAT: Campos inundáveis

DISTRIBUIÇÃO: Guiana, Suriname, Paraguai e Brasil: AM, AP, DF, GO, MA, MG, MT, PR, RR, RS

ESPÉCIE: Gongora nigrita Lindl. MG 66.940

HABITAT: Floresta ribeirinha e Igapó

DISTRIBUIÇÃO: Guianas, Brasil: MA, PA

ESPÉCIE: Gongora quinquenervis R \& Pav. MG 147.013

HABITAT: Floresta ribeirinha e igapó DISTRIBUIÇÃO: Do México ao Brasil, Sul do Peru e Trinidad. Brasil: AC, AM, AP, MA, MT, PA, RO, RR

ESPÉCIE: Habenaria obtusa Lindl. MG 145.532

HABITAT: Campos inundados

DISTRIBUIÇÃO: Paraguai, Suriname, Brasil: GO, MA, MG
ESPÉCIE: Habenaria pratensis (Lindl.) Rchb.f. MG 19.345

HABITAT: Campos inundados

DISTRIBUIÇÃO: Guianas, Brasil: BA, GO, MA, MT, SP

ESPÉCIE: Ionopsis satyrioides (SW) Rchb.f. MG 135.348

HABITAT: Epífita de floresta aberta e pomares cultivados

DISTRIBUIÇĀO: Brasil: MA, PA

ESPÉCIE: Ionopsis utricularioides (Sw.) Lindl. MG 138.909

HABITAT: Floresta de terra-firme, de galeria e de igapó

DISTRIBUIÇÃO: Desue Flórida e México até o Paraguai; Brasil: BA, GO, MA, MG, MT, PA,. PR, SP

ESPÉCIE: Lepanthes brasiliensis Pabst. HABITAT: Floresta ribeirinha DISTRIBUIÇÃO: AP, MA, PA

ESPÉCIE: Lepanthes helicocephala Rchb.f. MG 147.848

HABITAT: Floresta ribeirinha.

DISTRIBUIÇÃO: Venezuela, Guiana, Suriname e Brasil: MA

ESPÉCIE: Leucohyle brasiliensis Schltr. HABITAT: Floresta de terra-firme.

DISTRIBUIÇÃO: Brasil: AP, MA, MT, PA, RO

ESPÉCIE: Leucohyle mutica (Rchb.f. \& W.) Schltr.

HABITAT: Floresta ribeirinha.

DISTRIBUIÇÃO: Trinidad, Venezuela, Suriname e Brasil: AP, MA, PA

ESPÉCIE: Lockhartia goyazensis Rchb. f. MG 22.269

HABITAT: Epifita de cerrado $\mathrm{c}$ floresta úmida 
DISTRIBUIÇÃO: Bolivia e Brasil: AP, BA, ES, MA, PA, RJ, RS, SC, SP

ESPÉCIE: Lockhartia lunifera $\mathrm{Rchb}$. $\mathrm{f}$. MG 106.358

HABITAT: Floresta úmida.

DISTRIBUIÇÃO: Brasil: AP, BA, ES, MA, PA, RJ, RS, SP, SC

ESPÉCIE: Macradenia rubescens Barb. Rodr. HABITAT: Floresta ribeirinha e floresta de galeria

DISTRIBUIÇÃO: Brasil: AM, MA, PA

ESPÉCIE: Maxillaria alba (Hook.) Lindl. MG 138.857

HABITAT: Floresta ribeirinha e floresta úmida DISTRIBUIÇÃO: Desde GuatemaIa, Honduras e Indias Ocidentais, da Colômbia até o Brasil: AM, GO, MA, MT, PA

ESPÉCIE: Maxillaria camaridii Rchb.f. MG 145.505

HABITAT: Floresta de terra-firme, floresta ribeirinha, igapó, campina

DISTRIBUIÇÃO: Costa Rica, Guatemala, Honduras, Panamá, América do Sul Tropical, Indias Ocidentais, Brasil: MA, PA

ESPÉCIE: Maxillaria rufescens LindI. MG 127.049

HABITAT: Floresta de terra-firme e campina

DISTRIBUIÇÃO: Desde as Guianas pelo Brasil Oriental: AC, AM, BA, ES, GB, MA, MG, PR, RJ, RS, SP, SC

ESPÉCIE: Maxillaria superflua Rchb.f. MG 148.451

HABITAT: Floresta de terra-firme, Floresta ribeirinha, igapó e campina

DISTRIBUIÇÃO: Guianas e norte do Brasil: AM, MA, MT, PA

ESPÉCIE: Maxillaria uncata Lindl. MG
135.358

HABITAT: Epifita de floresta úmida das margens dos rios

DISTRIBUIÇÃO; Brasil: AM, PA, MA, RO, RR.

ESPÉCIE: Notylia wullschlaegeliana Focke. MG 135.344

HABITAT: Floresta ribeirinha, floresta de galeria e campo rupestre

DISTRIBUIÇÃO: Brasil: AM, MA, PA

ESPÉCIE: Octomeria grandiflora Lindl. MG 147.827

HABITAT: Floresta de terra-firme, floresta ribeirinha, igapó e campina.

DISTRIBUIÇÃO: Brasil: AM, MA, PA, RR.

ESPÉCIE: Oeceoclades maculata (Lind.) Lindl.

HABITAT: Floresta higrófila, floresta de capoeira, floresta de várzea, floresta de terra-firme, caatinga arenosa.

DISTRIBUIÇÃO: África tropical, Venezuela, Paraguai, Argentina, Peru e Trinidad, em todo Brasil

ESPÉCIE: Oncidium baueri Lindl. MG 105.502

HABITAT: Floresta ribeirinha.

DISTRIBUIÇÃO: Do México ao Panamá, da Venezuela a Bolivia, Índias Ocidentais, Africa tropical, Paraguai, Peru, Trinidad e Brasil: AP, ES, MA, MG, PA, RJ, SP

ESPÉCIE: Oncidium cebolleta (Jacq.) Sw. MG 127.744

HABITAT: Epifita, às vezes latofitica, em igapó e outros ambientes

DITRIBUIÇÃO: Brasil: AC, AM, BA, GO, MA, MG, MT, PA, PE, PI, RO, RR, TO.

ESPÉCIE: Oncidium fuscopetalum (Hoehne) Garay. MG 135.343

HABITAT: Cerrado

DISTRIBUIÇÃO: Brasil: GO, MA, MT 
ESPÉCIE: Oncidium macropetalum Lindl. MG

HABITAT: Cerrado

DISTRIBUIÇÃO: Paraguai e Brasil: DF, GO, MA, MG, MT, SP

ESPÉCIE: Oncidium morenoi Dodson \& Luer. MG 108.083

HABITAT: Floresta de terra-firme, floresta ribeirinha e cerrado

DISTRIBUIÇÃO: Bolivia, Brasil: AC, AM, MA, PA, RO, RR, TO

ESPÉCIE: Oncidium nanum Lindl. MG 138.867

HABITAT: Epifita de igapó, campina e floresta de galeria

DISTRIBUIÇÃO: Brasil: AC, AM, MA, PA, TO, RO, RR

ESPÉCIE: Orleanesia amazonica Barb. Rodr. HABITAT: Floresta de igapó, floresta ribeirinha, floresta de terra-firme DISTRIBUIÇÃO: Brasil: AM, MA, PA

ESPÉCIE: Orleanesia yauaperyensis Barb. Rodr. MG 138.884

HABITAT: Vegetação de cerrado.

DISTRIBUIÇÃ̃O: Brasil: AM, GO, MA, MT, PA, TO

ESPÉCIE: Ornithocephalus gladiatus Hook. MG 141.270

HABITAT: Floresta de terra-firme.

DISTRIBUIÇÃO: Do México até o

Panamá, Antilhas, Bolivia, Colômbia, Guianas, Peru, Trinidad, Venezuela e Brasil: AM, GO, MA, PA

ESPÉCIE: Peristeria guttata Knowl. MG 139.107

HABITAT: Floresta de terra-firme

DISTRIBUIÇÃO: Colômbia, Venezuela, Guianas, Brasil: AM, PA, MA
ESPÉCIE: Pleurothallis modesta Cogn. MG 139.021

HABITAT: Floresta de terra-firme

DISTRIBUIÇÃO: Brasil: BA, MA, PA, PR, RJ, SP

ESPÉCIE: Pleurothallis pruinosa Lindl. MG 149.895

HABITAT: Floresta ribeirinha

DISTRIBUIÇÃO: América Central, Índias Ocidentais, Guianas, Venezuela, Brasil: AP, MA, PA

ESPÉCIE: Polystachya concreta (Jacq.) Garay et Sweet. MG 145.503

HABITAT: Habita todos os tipos de ecossistemas

DISTRIBUIÇÃO: Brasil: toda região amazônica.

ESPÉCIE: Polystachya foliosa (Lindl.) Rchb. f. MG 139.860

HABITAT: Epifita de todos os habitats DISTRIBUIÇÃO: Brasil: AM, MA, PA, RR, TO

ESPÉCIE: Polystachya stenophylla Schltr. MG 138.887

HABITAT: Epifita de igapó e de floresta úmida

DISTRIBUIÇÃO: Brasil: AM, MA, PA

ESPÉCIE: Psygmorchis glossomistax (Rchb. f.) Dodson \& Dressler. MG 5.688 HABITAT: Floresta ribeirinha, floresta de terra-firme

DISTRIBUIÇÃO: Colômbia, Equador, Peru, Venezuela, Brasil: AC, AM, BA, ES, MA, PA, RJ

ESPÉCIE: Psygmorchis pusilla (L.) Dodson \& Dressler. MG 108.037

HABITAT: Floresta ribeirinha, floresta de terra firme e caatinga arenosa.

DISTRIBUIÇÃO: América Central, 
Colômbia, Peru, Bolivia, Venezuela, Guiana Francesa, Brasil: AM, AP, BA, GO, MA, MT, RO, RJ,

ESPÉCIE: Quekettia microscopica Lindl. MG 515

HABITAT: Floresta de terra-firme, floresta ribeirinha, campina

DISTRIBUIÇÃO: Brasil: AM, MA, PA

ESPÉCIE: Reichenbachanthus reflexus (Lindl.) Brade MG 145.550

HABITAT: Floresta úmida, campina e igapó.

DISTRIBUIÇÃO: Brasil: AM, AP, MA, PA

ESPÉCIE: Rodriguezia lanceolata Ruiz et Pav. MG 104.479

HABITAT: Floresta de terra-firme, floresta ribeirinha, campina, campo rupestre.

DISTRIBUIÇÃO: América do Sul Tropical, Brasil: AM, AP, MA, MT, PA

ESPÉCIE: Sacoila lanceolata (Aubl.) Garay. MG 146.505

HABITAT: Floresta de terra-firme, cerrado DISTRIBUIÇÃO: Brasil: DF, GO, MA, MG, MT, PA, PE, PR, RJ, RS, SC, SP

ESPÉCIE: Scaphyglottis amethystina (Rchb.f.) Schltr. MG 138.880

HABITAT: Floresta de terra-firme, floresta ribeirinha, igapó, campina, campo rupreste e cerrado

DISTRIBUIÇÃO: Colômbia, Costa Rica, Guatemala, Nicarágua, Panamá, Suriname, Venezuela, Brasil: AC, AM, AP, MA, MT, PA, RO, RR, TO

ESPÉCIE: Scaphyglottis modesta Schltr. HABITAT: Floresta de terra-firme DISTRIBUIÇÃO: Brasil: AM, MA, PA

ESPÉCIE: Scaphyglottis sickii Pabst. MG 147.837

HABITAT: Epifita de floresta aberta
DISTRIBUIÇÃO: Brasil: AM, AP, MT, MA, PA, PE

ESPÉCIE: Schomburgkia gloriosa Rchb.f. MG 141.265

HABITAT: Epífita de igapó, cerrado e floresta aberta

DISTRIBUIÇÃO: Brasil: AM, MA, AM, MT, PA, RO, RR,TO

ESPÉCIE: Sobralia macrophylla Reichb.f.. MG 147.826

HABITAT: Floresta ribeirinha e campina DISTRIBUIÇÃO: Costa Rica, Colômbia, Venezuela, Panamá, Brasil: AM, AP, PA

ESPÉCIE: Sobralia sessilis Lindl. MG 110.729

HABITAT: Floresta ribeirinha, igapó, campina.

DITRIBUIÇÃO: Venezuela, Guianas e Brasil: AC, AM, AP, MA, MG, PA, RR

ESPÉCIE: Solenidium lunatum (Lindl.) Krzl. MG 126.790

HABITAT: Floresta de terra-firme e campina DISTRIBUIÇẢO: Venezuela, Guiana c Brasil: GO, MA, MT, PA

ESPÉCIE: Stanhopea grandiflora (Lodd.) Lindl. MG 141.268

HABITAT: Floresta de terra-firme e floresta ribeirinha

DISTRIBUIÇÃO: Trinidad, Venezuela, Guianas, Colômbia e Brasil: AM, MA, PA

ESPÉCIE: Trigonidium acuminatum Batem. MG 43.211

HABITAT: Floresta ribeirinha.

DISTRIBUIÇÃO: Brasil: DF, MA, RJ

ESPÉCIE: Trigonidium tenue Lodd. MG 149.860

HABITAT: Floresta de terra-firme, floresta ribeirinha, igapó, campina, campo rupestre e cerrado 
DISTRIBUIÇÃO: Venezuela, Brasil: AM, AP, BA, ES, MA, PA, PE, RO

\section{ESPÉCIE: Vanilla palmarum Lindl.}

HABITAT: Floresta de terra-firme e floresta ribeirinha

DISTRIBUIÇÃO: Venezuela e amplamente no Brasil

ESPÉCIE: Zigosepalum labiosum (L.C. Rich.) Garay. MG 148.448

HABITAT: Floresta ribeirinha e de igapó. DISTRIBUIÇÃO: Colômbia, Venezuela, Guiana Francesa e Brasil: AP, AM, MA $\mathrm{PA}, \mathrm{RO}$

\section{AGRADECIMENTOS}

Ao Departamento de Botânica do Museu Paraense Emílio Goeldi, sede do projeto científico e onde estão depositadas as coleções de referência; aos órgãos de fomento à pesquisa que nos concederam apoio financeiro para as excursões ou bolsas de estudo: CAPES, CNPq, FNMA,FBMM e às empresas ou órgãos que têm nos apoiado com logística de campo: Cia Vale do Rio Doce, PETROBRAS, ELETRONORTE, ALBRÁS, IBAMA, Primeira Comissão Demarcadora de Limites; ao Royal Botanic Gardens of Kew-UK, pela concessão do estágio em seu Herbário e Biblioteca; aos colegas que colaboraram para a realização deste trabalho, entre estes, os alunos Antônio Elielson da Rocha, Edieli Serra Pena, Eliza Miki, Francisco Plácido, Silvana Helena N. Monteiro;
Ruy e aos demais companheiros, não citados mas que muito colaborarm conosco, principalmente dando apoio moral através da confiança depositada.

\section{Bibliografia citada}

Cogniaux, A. 1896 Orciidaceae. In: Martius, K.F.P. von, ed. Flora brasiliensis. Monachii, Frid. Fleisher. v. 3, fasc. 4. 672p.

Cogniaux, A. 1902 Orchidaceae. In: Martius, K.F.P. von, ed. Flora brasiliensis. Monachii, Frid. Fleischer. v. 3, fasc. 5. 664p.

Cogniaux, A. 1906 Orchidacede. In: Martius. K.F.P. von, ed. Flora brasiliensis. Monachii, Frid. Fleischer. v. 3, fasc. 6. 604p.

Dressler, R.L. 1993 Phylogeny and Classification of the Orchid Family. Cambridge University Press. 314p.

Dunsterville, G.C.K.; Garay, L.A. 1972-76 Venezuelan orchids illustrated. Amsterdam, Drukkerij Holland N.V. 5 v. 1055p.

Hoehne, F.C. 1942 Orchiaacene. Ir: Flora Brasilica. São Paulo, Secretaria da Agricultura/Instituto de Botânica. t. 12, v: 6 .

Hoehne, F.C., 1945 Orchidaceae. In: Flora Brasílica. São Paulo, Secretaria da Agricultura/ Instituto de Botânica, t. 12, v. 2, 389p.

Hoehne, F.C., 1949 lconografia de Orchidaceas do Brasil. São Paulo, Secretaria da Agricultura. 301p.

Hoehne, F.C., 1953 Orchidaceae, In: Flora Brasilica. Sào Patilo, Socretaria da Agricultura Instituto de Botânica. t. 12, v. 7, 397p.

Pabst, G.F.J.; Dungs, F. 1975 Orchidaceae Brasiliensis I. Hildesheim, Brucke-Kurt Schmersow. 408p.

Pabst, G.F.J.; Dungs, F. 1977 Orchidaceae Brasiliensis II. Hildesheim, Brucke-Kurt Schmersow. 418p. 